\title{
Acompanhamento da escrita coletiva a distância: tecnologia para apoiar a ação docente
}

\author{
Alexandra Lorandi Macedo - UFRGS - alorandimacedo@gmail.com \\ Patricia Alejandra Behar - UFRGS - pbehar@terra.com.br \\ Eliseo Berni Reategui - UFRGS - eliseoreategui@gmail.com
}

\begin{abstract}
Resumo
Este artigo destaca a construção e a contribuição da Rede de Conceitos, uma ferramenta desenvolvida com o objetivo de apoiar a prática pedagógica do professor para $\mathrm{o}$ acompanhamento da produção de textos no Editor de Texto Coletivo (ETC). Foi a partir de diferentes práticas desenvolvidas neste Editor que se percebeu a dificuldade que o docente tem para administrar o alto e permanente volume de dados advindos das produções textuais coletivas apoiadas pelos recursos digitais, neste caso o ETC. A Rede foi desenvolvida através da tecnologia de mineração de textos e, a partir dos seus resultados, favorece a otimização do tempo de leitura do professor diante do alto e permanente volume de dados gerados na produção coletiva.
\end{abstract}

Palavras-chave: Produção textual coletiva, Mineração de Texto.

\begin{abstract}
This study highlights the construction and the contribution of the Concepts Network, a tool developed with the aim of supporting the teacher's pedagogical practice in monitoring the production of texts in the Collective Text Editor (ETC). It was from the different practices developed in this Editor, that the difficulty the teacher faces was noticed, namely in managing the high and continuous volume of data resulting from the collective textual productions, supported by digital resources, in this case the ETC. The Network was developed through text mining technology and allows the optimization of the teacher's reading time in the face of the high and constant volume of data generated in collective production.
\end{abstract}

Keywords: Text Mining, Collective textual production.

\section{Introdução}

Este artigo destaca a construção e a contribuição da Rede de Conceitos, uma ferramenta desenvolvida com o objetivo de apoiar a prática pedagógica do professor para o acompanhamento da produção de textos no Editor de Texto Coletivo - ETC (disponível em: www.nuted.ufrgs.br/etc2). Foi a partir de diferentes práticas desenvolvidas neste Editor que se percebeu a dificuldade que o docente tem para administrar o alto e permanente volume de dados advindos das produções textuais coletivas apoiadas pelos recursos digitais, neste caso o ETC (BEHAR et al., 2006).

Atualmente, a construção coletiva tem ganhado destaque como uma alternativa eficaz para acompanhar e dar conta do exponencial crescimento e proliferação da informação apoiada, principalmente, nos recursos digitais. Aqui, os alunos aprendem em um processo interativo, coletivo, que pode favorecer uma aprendizagem qualitativamente melhor do que a desenvolvida numa prática individual. Assim, a interação e a colaboração alcançam seu objetivo por proporcionar a troca de ideias, dúvidas e diferentes pontos de vista, privilegiando sucessivas construções. 
Em paralelo, o contexto educacional apoiado no virtual comporta objetos que transitam entre os grupos, comporta memórias compartilhadas, hipertextos comunitários para a formação de coletivos inteligentes (Lévy, 1993). Nessa perspectiva, o suporte tecnológico pode vir a resgatar a atividade do sujeito como construtor de seu conhecimento e das interações sociais. O mesmo autor destaca ainda que tais interações devem ser concebidas como um processo de crescimento, de diferenciação, de ramificação e retomada mutual de singularidades em oposição à ideia de uma fusão de inteligências individuais. Assim, o que se quer é valorizar os diferentes olhares, as diferentes perspectivas e cruzá-las em prol de um interesse comum. Porém, todo esse movimento é feito, predominantemente, através da escrita. Logo, o foco do problema identificado junto aos docentes que propõem práticas de textos coletivos é a quantidade de tempo necessária para acompanhar o alto volume de dados gerados no processo de construção da escrita. Por isso, o desafio foi encontrar uma solução capaz de retornar ao professor dados relevantes sobre o texto, sem exigir deste, inúmeras releituras da produção textual. Foi então que, a partir de inúmeras análises e investigações, optou-se pela tecnologia de Mineração de Texto para o desenvolvimento da Rede de Conceitos (MACEDO, 2010).

\section{Mineração de Texto: desenvolvimento e aplicação da Rede de Conceitos}

A Mineração de Textos permite que sejam extraídos e relacionados os principais termos tratados em um texto (FELDMAN, 2007). Com base na extração e relacionamento dos mesmos, a ferramenta apresenta um grafo que possibilita a visualização do conteúdo extraído. Essa é a tecnologia que deu suporte para o desenvolvimento da Rede de Conceitos.

O processo que gera a Rede de Conceitos compreende como primeira etapa a análise léxica, onde a produção textual é desmembrada palavra por palavra. A seguir, todos os conceitos extraídos são submetidos à análise estatística. Nesse momento, com base nos dados estatísticos, é criada uma base de conceitos, que posteriormente auxiliará na construção da Rede. O passo seguinte consiste na remoção de palavras que não agregam significado ao texto, tais como: artigos, conjunções dos verbos ser, estar, ter e haver, bem como pronomes. Esse processamento baseou-se no método utilizado por Schenker (2003). De posse da análise estatística, cria-se a Rede de Conceitos. Nessa atividade, parâmetros como frequência absoluta e tamanho do conceito (se simples ou composto) são analisados.

A partir do resultado gerado pela Rede de Conceitos, iniciou-se o processo de implementação e de validação da ferramenta no Editor de Texto Coletivo. A Figura 1 mostra a interface da Rede sobreposta à interface do Editor. Foi então que se delinearam as primeiras aproximações entre os resultados da Rede e os textos de origem, com o objetivo de responder às demandas identificadas no estudo. 


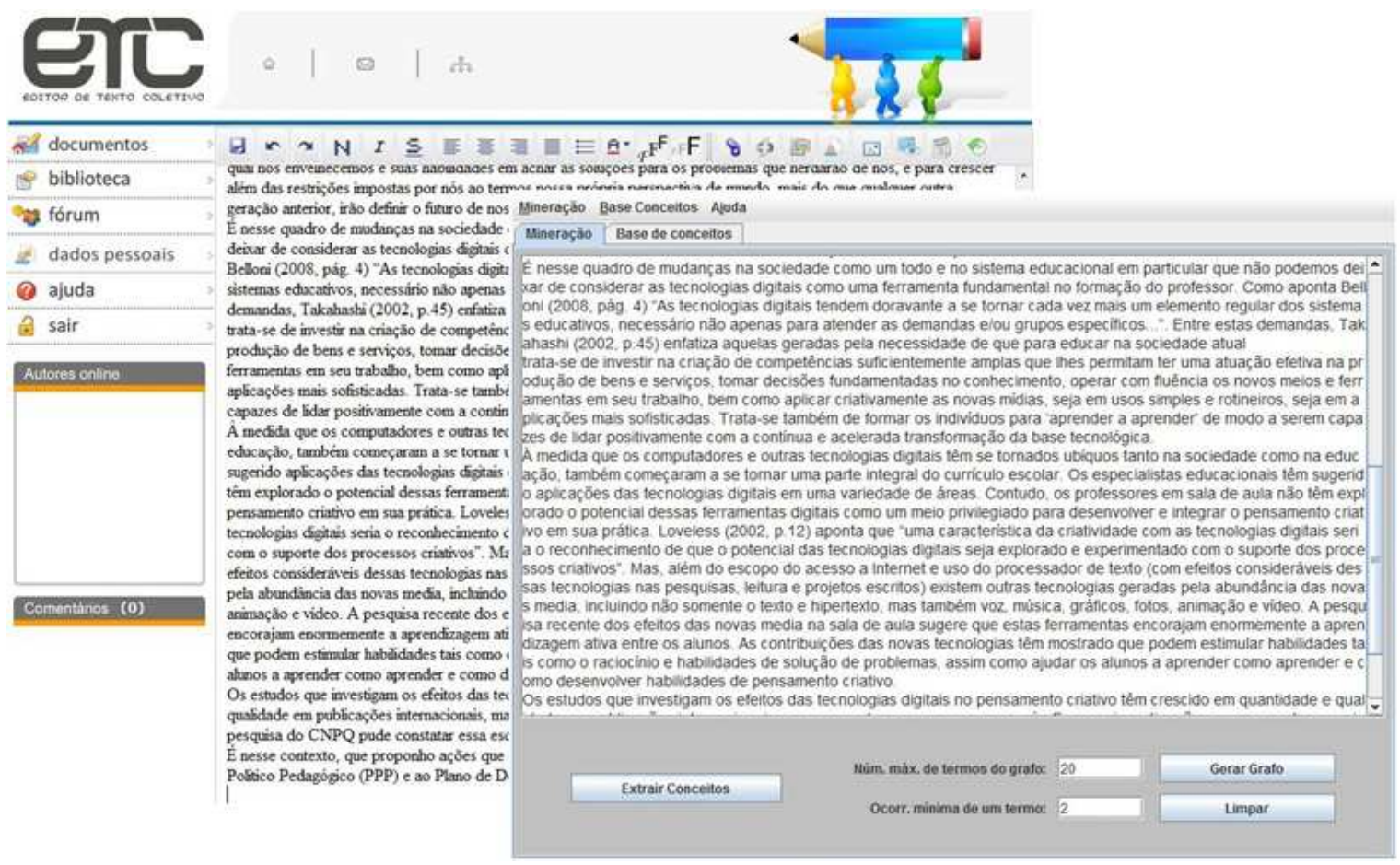

Figura 1 - Interface da Rede de Conceitos sobreposta à interface do ETC

Participaram deste estudo, na fase de implementação, 58 estudantes de diferentes disciplinas/cursos oferecidos pela Universidade Federal do Rio Grande do Sul. São eles:

* Curso de Extensão Universitária "Competências para o Trabalho em Equipe" curso aberto à comunidade.

* Disciplina "Oficinas Virtuais de Aprendizagem" - desenvolvida no Programa de Pós-Graduação em Educação e Pós-Graduação em Informática na Educação e, em outra turma, no curso de Pós-Graduação do Doutorado Interinstitucional (DINTER).

* Disciplina "O computador na educação" - desenvolvida no curso de graduação em Pedagogia na modalidade a distância (PEAD).

$\mathrm{Na}$ primeira etapa de análise dos dados foram agrupadas as Redes que mostraram semelhança na apresentação dos termos e separadas em dois grupos: um com maior incidência de termos soltos, outro com maior incidência de termos relacionados/conectados entre si. A análise da Rede em paralelo com a leitura dos textos mostrou que, do primeiro grupo, fazem parte os textos que necessitam aprimoramento, uma vez que a falta de conexão advém das produções sem encadeamento e sequência lógica. Já do segundo grupo, com incidência de termos relacionados entre si, fazem parte textos com coerência, sequência e lógica no desenvolvimento. O objetivo dessa prática foi identificar quais eram os indicadores retornados pela ferramenta. E, com base neles, notou-se a importância que a Rede pode ter na prática diária do professor, já que, por não precisar fazer inúmeras leituras do texto, aumenta seu tempo de interação com os alunos.

Em posse dos dados coletados, foram construídos apontamentos para prática pedagógica com o objetivo de oferecer suporte ao professor que utilizar a ferramenta. Tais apontamentos são desenvolvidos na próxima seção. 


\section{Rede de conceitos: perspectivas educacionais}

Esta seção tem por objetivo descrever algumas aplicações da Rede de Conceitos feita em contextos educacionais, apontando os resultados obtidos e, a partir destes, indicando possibilidades de práticas pedagógicas.

Vale ressaltar que este estudo não tem a pretensão de criar uma regra de análise para as Redes e nem de estabelecer práticas pedagógicas únicas. $\mathrm{O}$ objetivo é apontar possibilidades e oferecer condições para que cada professor analise o material de acordo com suas necessidades e especificidades do grupo de alunos.

Considerando a Rede de Conceitos em destaque na Figura 2, mesmo sem ter lido o texto de origem, é possível perceber, a partir dos conceitos exibidos no grafo, que a mesma aborda eixos como: aprendizagem, computador, interatividade, novos rumos, conhecimento, cooperação, troca, tecnológicas, escolas, entre outros. Assim, aponta para um cenário que privilegia uma abordagem onde a educação e a tecnologia estão em relação. Ainda, na análise da Rede, é possível presumir que tal abordagem perpassou sobre a questão da formação docente. Tal afirmação é feita com base nos conceitos que estão relacionados e exibidos na parte inferior da mesma, conforme consta na Figura 02. Ali, conceitos como: educação, realidade, formas, tecnologia, processo, formação, professor e reflexão, podem indicar um desdobramento no texto, que tratou da importância da formação docente em relação ao uso da tecnologia na educação.

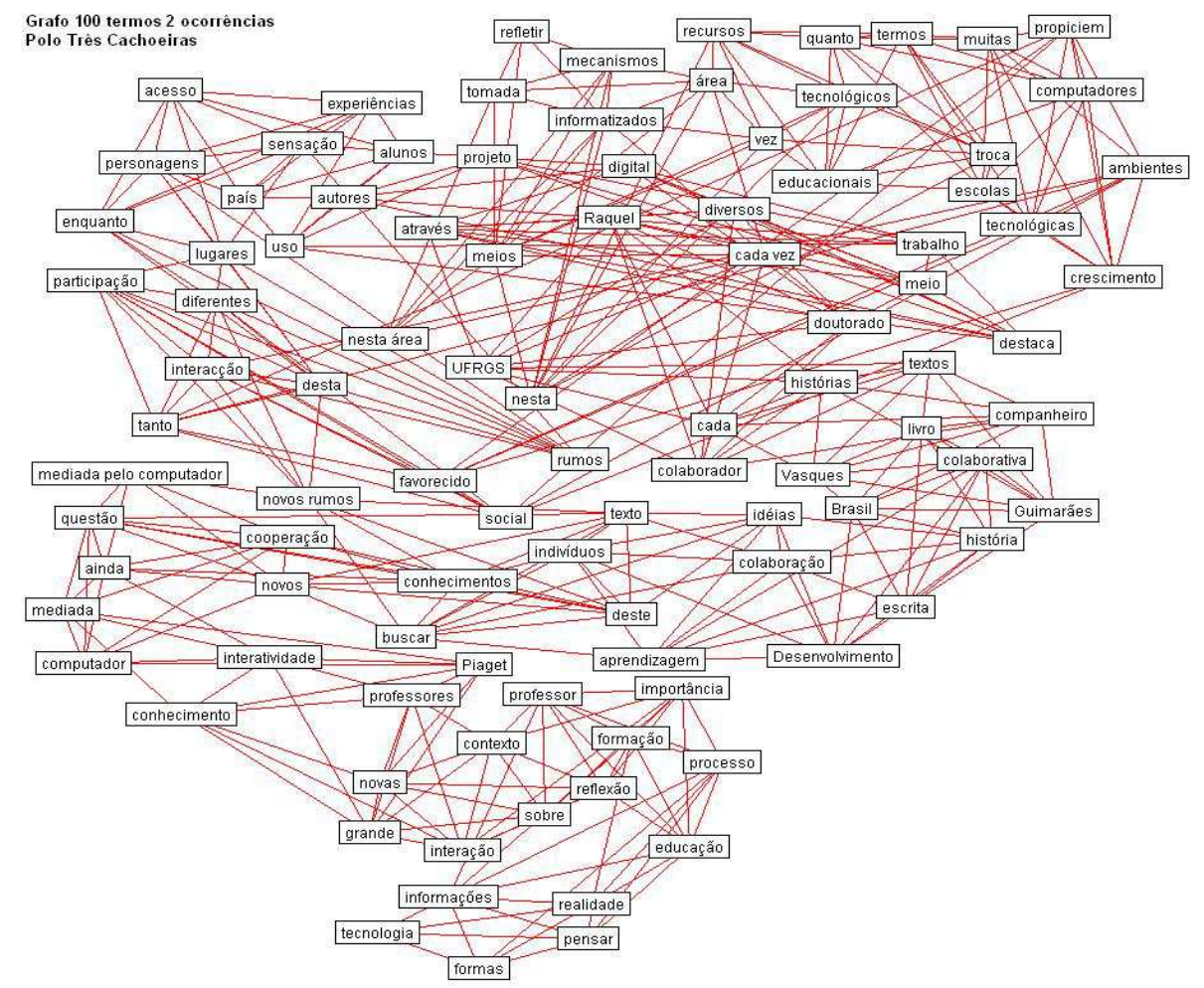

Figura 2 - Rede de Conceitos - Identificação do tema 
Com base nessas considerações, os extratos destacados na Tabela 1, visam aproximar a relação entre o texto e a Rede de Conceitos, a fim de conferir as hipóteses levantadas.

Tabela 1. Rede de Conceitos - Identificação do tema

A tecnologia tem se manifestado amplamente em hábitos, necessidades, formas de pensar a realidade, de obter e trocar informações, de comunicar-se ou utilizar a linguagem. Se este processo for relacionado com a educação e a formação do professor, a importância dessas áreas estará em evidência para a reflexão sobre esse fenômeno. A necessidade de compreender este processo remete ao contexto da interação como transformação e novas concepções dialéticas.

Há muitas razões para investir em computadores nas escolas. $\mathrm{O}$ aluno sem computador está em franca desvantagem, tanto em termos de conhecimento de tecnologia quanto em termos de trabalho cooperativo e colaborativo.

Trata-se de uma questão de responsabilidade social oferecer computadores a esses jovens nas escolas que proporcionariam variedade de textos, modos de comunicação...

É reconhecido pelos professores, diretores e autoridades educacionais que o computador é um precioso colaborador para a comunicação, à troca de idéias e informações de atividades em comum.

O grande crescimento da comunicação e conseqüentemente da troca de informações entre os indivíduos por meios cada vez mais informatizados, transforma os meio de comunicação em recursos e mecanismos de aprendizagem.

Estes meios, tanto os informatizados quanto os não-informatizados, estimulam novas formas de pensamento e de comunicação que contribuem para análise, reflexão e tomada de novos rumos.

Diante do exposto destaca-se que, quando a análise da Rede de Conceitos permite identificar o tema desenvolvido, é porque os autores conseguiram eleger um eixo temático e desenvolveram uma produção textual que contempla, em diferentes momentos da escrita, o assunto que se propuseram ou foram desafiados a desenvolver. Frente à esta constatação destaca-se que o resultado da Rede tem estreita relação com a estrutura e o conteúdo desenvolvido no texto. Sendo assim, se a Rede não dá indícios do tema abordado, isso pode ser um indicativo de que a produção textual não deu foco ao assunto que se propôs desenvolver. Frente a tal constatação, sugere-se atenção em relação às trocas. Sabe-se que a Rede de Conceitos é resultado das trocas sociais no Editor de Texto Coletivo quando da construção de um texto. Assim, sugere-se que a prática pedagógica privilegie experiências estimuladoras de decisão e responsabilidade. Além disso, que dispare aos alunos desafios e questionamentos que favoreçam tanto o exercício da tomada de decisões, quanto a busca por recursos materiais que possam beneficiar novas construções e permitam aos alunos avançar qualitativamente na produção. Destaca-se ainda que, num cenário onde a partir da Rede de Conceitos é possível identificar o tema desenvolvido, o que indica que a produção escrita dá foco ao assunto proposto, o professor poderá utilizar as informações como base para investigar o nível de conhecimento dos alunos sobre o tema. Com base nisso, o docente poderá proporcionar materiais e discussões que viabilizem o aprofundamento teórico sobre o assunto. Assim, tais condições podem alavancar o conhecimento e relacionamento do V. $10 \mathrm{~N}^{\circ} 1$, julho, 2012 
tema em questão com outras vertentes, favorecendo novos desdobramentos, ampliando o conhecimento, bem como aprimorando as trocas sociais que apoiam a escrita coletiva de texto.

Com base nas relações feitas, salienta-se que uma prática pedagógica que incentive e crie condições favoráveis à pesquisa, leitura, análise, discussão interindividual e produção de novos conhecimentos, poderá proporcionar o desenvolvimento da autonomia, formando um sujeito com espírito crítico, com iniciativa para ir em busca de elementos que atendam suas necessidades (aprender a aprender), articulando e confrontando suas perspectivas com outras, de outros sujeitos que pensam e argumentam sob diversos pontos de vista.

Em outro foco de análise da Rede de Conceitos, notou-se que a estrutura dela tem relação direta com a qualidade da produção textual, isto é, as Redes, de certa forma, 'denunciam', por exemplo, textos com necessidade de aprofundamento teórico ou com justaposição de parágrafos. Algumas, ao longo da análise, chamaram atenção pela falta de encadeamento entre os termos que a compõem. Exemplo disso é a Rede representada pela Figura 3.

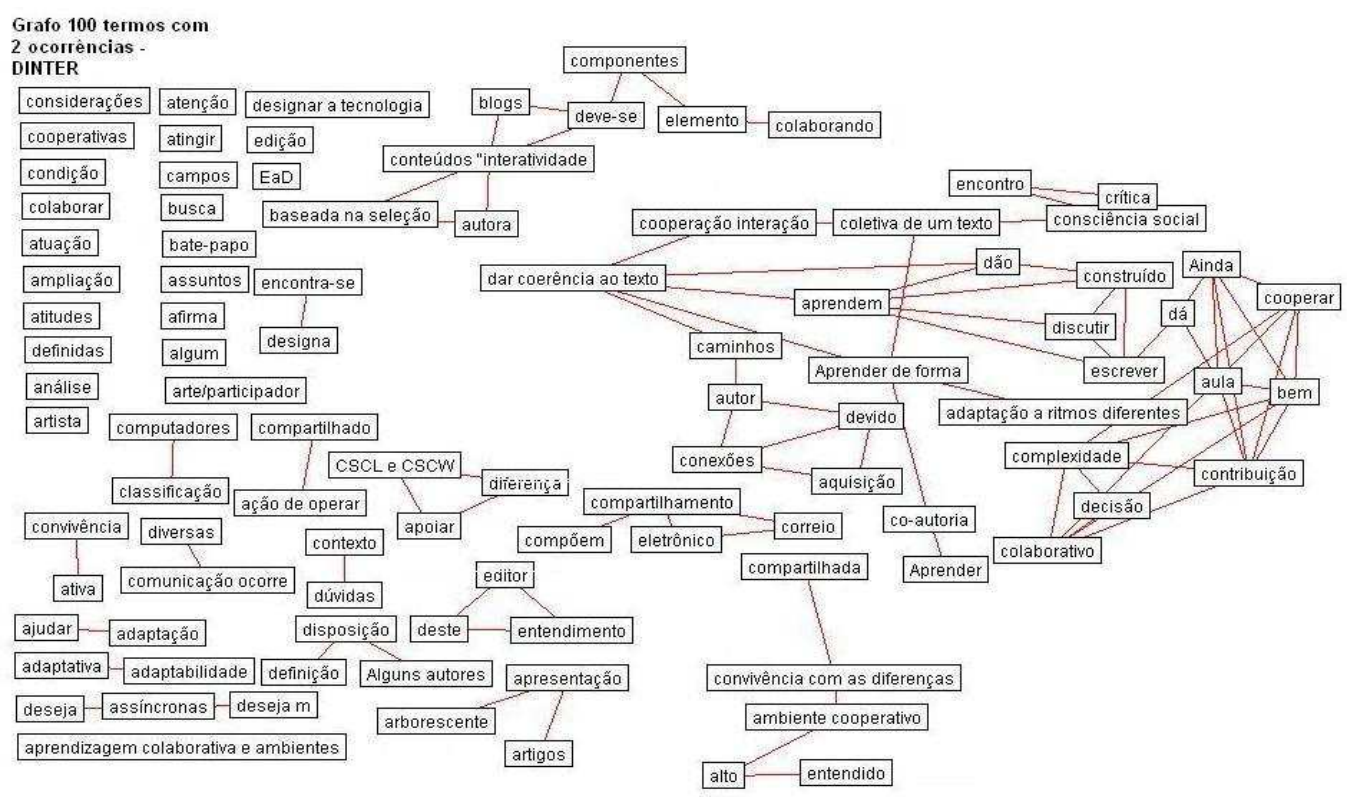

Figura 3 - Rede de Conceitos - Incidência de termos soltos

Nessa Rede é possível perceber uma quantidade significativa de termos isolados ou relacionados com apenas um outro. Também é significativo o número de pequenos conjuntos de termos. Porém, mesmo com tais características, neste caso, a leitura da Rede permite identificar o tema desenvolvido na produção textual. Conceitos como: colaborando, ambiente cooperativo, compartilhado, aprendizagem colaborativa e ambientes, CSCL (Computer Supported Collaborative Learning) e CSCW (Computer Supported Collaborative Work), coautoria, discutir, escrever, entre outros, podem indicar, por exemplo, uma produção textual com foco nos processos coletivos.

Ao longo desta produção textual os conceitos são tratados, cada qual, em uma seção específica. A abordagem é feita de forma descritiva e permeada de citações e referências teóricas. Nesse texto, as seções da produção não se relacionam entre si, V. $10 \mathrm{~N}^{\mathrm{o}}$ 1, julho, 2012 
assim, cada conceito é considerado de forma isolada, dentro do seu próprio eixo. A penúltima seção trata da descrição da experiência do grupo na construção do próprio texto e traz considerações sobre o uso do ETC. Entende-se que este poderia, por exemplo, ser um momento propício para retomar os conceitos acerca da aprendizagem cooperativa, objetivo inicial do texto, e refletir se tais conceitos perpassaram a experiência da produção textual coletiva a distância, feita por este grupo. No entanto, a seção descreveu de forma geral os procedimentos realizados e seguiu com as considerações finais, onde produziu um encerramento vinculado à importância dos processos coletivos e do auxílio da internet para a aprendizagem cooperativa. Diante disso, este estudo entende que a Rede de Conceitos correspondente a esse texto reflete bem a estrutura do mesmo. Tal Rede exprime uma significativa parcela de conceitos isolados, assim como foram tratados na produção textual.

Nas produções textuais em que os indivíduos não reconhecem o sentido das palavras empregadas, não compartilham proposições que fundamentam a troca de ideias, ou ainda, não conservam os acordos feitos, prejudicando assim a reciprocidade entre os participantes do grupo, as condições para a construção do texto ficam extremamente comprometidas. Desse cenário, resultam as Redes de Conceitos fragmentadas, compostas pela incidência de termos soltos. Por isso, proporcionar condições que favoreçam a leitura, a reflexão, novas construções e discutir essas perspectivas com os demais sujeitos é um exercício de aprendizagem. É nessa direção que este estudo acredita que deva estar apoiada a prática pedagógica que se preocupa em criar condições para favorecer interações balizadas nas condições de equilíbrio previstas por Piaget (1973).

Em paralelo, a análise das Redes apontou uma estrutura diferente da anterior, onde prevaleceram grupos de conceitos interligados, conforme destaca a Figura 4.

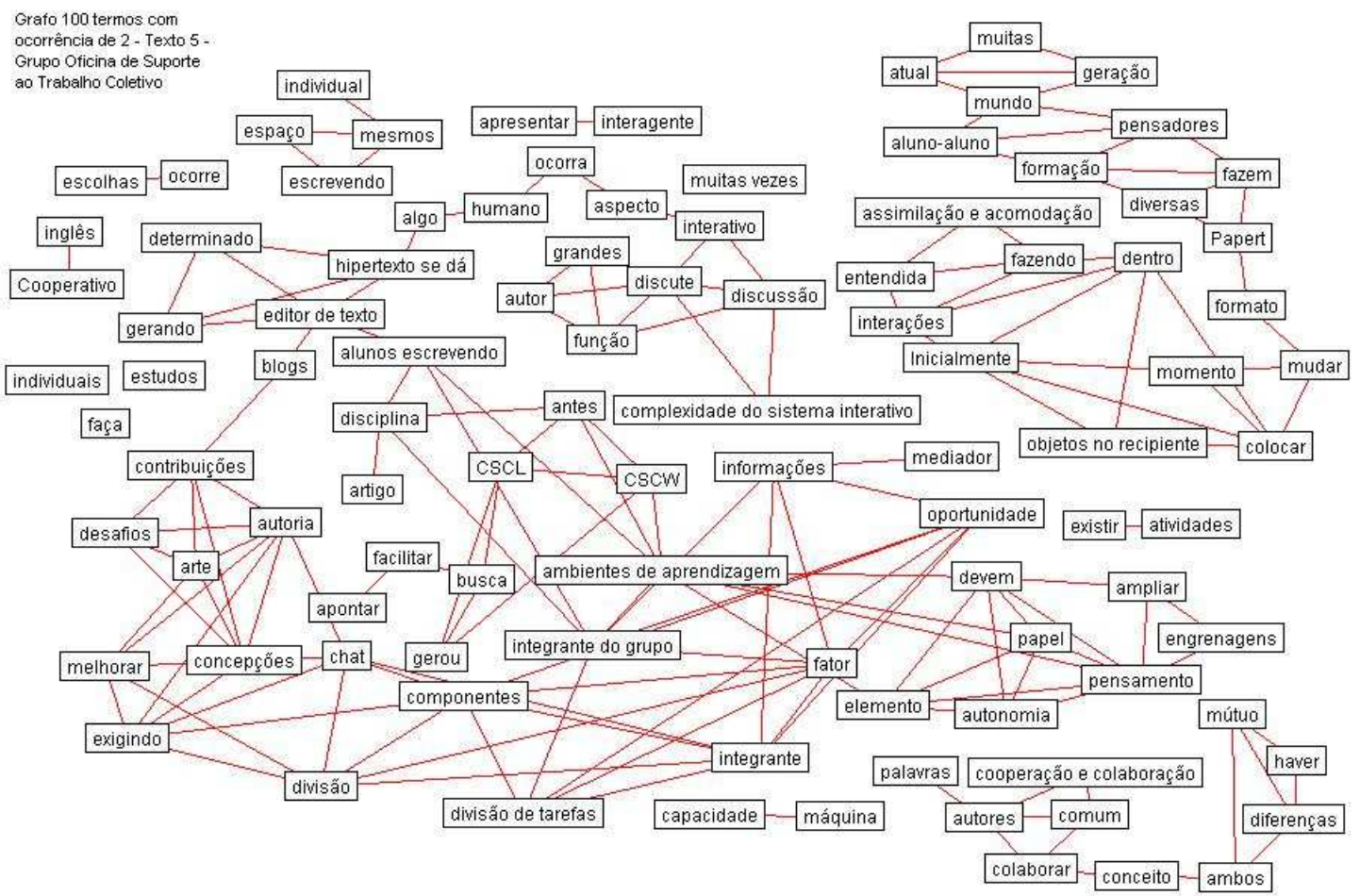

Figura 4 - Rede de Conceitos - Incidência de termos relacionados

V. $10 \mathrm{~N}^{\mathrm{o}} 1$, julho, 2012 
Os conceitos desta Rede, de forma geral, se complementam formando uma unidade temática. Por exemplo: editor de texto, ambientes de aprendizagem, blog, chat, divisão de tarefas, cooperação e colaboração, complexidade do sistema interativo, discussão, contribuições, autoria e desafios, são alguns dos conceitos que, mesmo não estando inteiramente interligados por arestas, complementam-se na indicação do tema central da produção textual.

Feita a análise da Rede de Conceitos, partiu-se para a leitura do texto, onde verificou-se que a introdução aponta os eixos que serão contemplados na produção textual, entre eles, o processo desenvolvido pelo grupo na atividade de EAD e conceitos como CSCL, CSCW, cooperação, colaboração, interação e aprendizagem, além de discussão sobre tecnologias na educação. A primeira seção trata da conceitualização dos termos CSCW, CSCL, cooperação e colaboração. Estes dois últimos conceitos introduzem o termo interação, que é definido, fundamentado e contextualizado, e apresentando em subseções eixos como: interação e hipertexto, interação e aprendizagem. Essa abordagem introduz o tópico que fala sobre interação e a escrita coletiva computacional e segue com foco no uso do ETC (Editor de Texto Coletivo) em relação ao processo do grupo e suas aplicações. A produção textual finaliza com considerações sobre o uso da tecnologia como possibilidade de apoio à aprendizagem.

Destaca-se que esse texto apresenta encadeamento, bom desenvolvimento, lógica na abordagem e boa argumentação. Diante disso, vale lembrar que o mais importante num processo de aprendizagem não é considerar somente o patamar que o sujeito conseguiu alcançar, mas analisar o processo percorrido por este sujeito, de onde partiu até onde conseguiu chegar (BECKER, 2001).

Nessa perspectiva, entende-se que o professor deve perceber o momento de intervir para favorecer a relação que o aluno deverá fazer entre o objeto de conhecimento e o nível de desenvolvimento que este se encontra naquele momento. Isso não pressupõe um método único de trabalho, ao contrário, entende-se que a prática pedagógica deve adotar diferentes formas de atuação para diferentes necessidades, tendo sempre como objetivo a construção do conhecimento do aluno. Em paralelo, o docente deve aprender a reconhecer e escolher ações que tenham chance de produzir resultados cognitivos, transformando, assim, as estruturas de conhecimento de cada sujeito (BECKER, 2001). Destaca-se que não é qualquer ação que proporciona transformações cognitivas, e sim uma ação significativa, que tenha sentido para o sujeito, que o faça pensar sobre o que fez e sobre o próprio pensamento. Daí parte a necessidade de a prática pedagógica estar inserida em um permanente processo reflexivo, que permita analisar as potencialidades dos recursos digitais e as intervenções necessárias para proporcionar o desenvolvimento da aprendizagem do aluno. Aliado a isso, o professor pode favorecer condições para que o aluno se dê conta "[...] de como age, tornando-se capaz de reproduzir sua ação corrigindo seus rumos, eliminando trajetos desnecessários ou criando trajetos novos, dirigindo-a para novos objetivos" (BECKER, 2003, p. 29).

\section{Considerações finais}

Este artigo teve por objetivo descrever o suporte oferecido pela Rede de Conceitos com vistas a apoiar a prática do professor que, diante das potencialidades dos recursos digitais, vê-se inserido num alto e permanente volume de dados gerados a 
partir das produções e das dinâmicas trocas coletivas. De forma objetiva, entende-se que as principais contribuições estão na significativa otimização do tempo de leitura exigido do professor para o acompanhamento da produção textual coletiva dos alunos. Como consequência, tem-se um melhor aproveitamento deste tempo para interação direta entre alunos e professor, o que pode proporcionar significativa qualificação no processo ensino-aprendizagem. Além disso, os indicadores evidenciados pela Rede de Conceitos podem auxiliar o professor a dar foco nas suas ações, agindo diretamente nas necessidades e potencialidades dos alunos. Tais condições podem ampliar as possibilidades de construção do conhecimento e a qualificação da produção escrita.

Por fim, destaca-se como possibilidade futura a aplicação da Rede em outras ferramentas, como por exemplo: fórum, histórico e chat, as quais permitem que se conheça o processo e as etapas de construção da produção textual. Nesse sentido, poderiam ser analisadas as discussões feitas sobre o tema da produção, bem como a participação de cada integrante em relação ao resultado final. Destaca-se também como possibilidade, que a Rede retorne os termos com links, que ao serem clicados, remetam para a parte do texto onde o conceito é contextualizado facilitando acesso para possíveis esclarecimentos sobre o termo empregado.

\section{Referências}

BECKER, F. Educação e Construção do Conhecimento. Artmed, Porto Alegre, 2001, Brasil.

BECKER, F. A origem do conhecimento e a aprendizagem escolar. Artmed, Porto Alegre, Brasil, 2003.

BEHAR, P. A. et al. Escrita Coletiva: o potencial de um Groupware via Web. RENOTE. Revista Novas Tecnologias na Educação. Porto Alegre, Brasil, vol. 4, no. 1, 2006.

FELDMAN R.; SANGER J. Text Mining Handbook. Inglaterra: Universidade de Cambridge, 2007.

LÉVY, P. As tecnologias da inteligência. Editora 34, Rio de Janeiro, Brasil, 1993.

MACEDO, A.L.. Rede de Conceitos: uma ferramenta para contribuir com a prática pedagógica no acompanhamento da produção textual coletiva. Tese de Doutorado. Universidade Federal do Rio Grande do Sul, Programa de Pós-Graduação em Informática na Educação, Porto Alegre, Brasil, 2010, 206 pp.

PIAGET, J. Estudos Sociológicos. Forense, Rio de Janeiro, Brasil, 1973.

SCHENKER, A. Graph-Theoretic Techniques for Web Content Mining. Tese (Doutorado em Ciência da Computação) - University of South Florida, 2003. 